\title{
Human Recognition in passive environment using Bidirectional associative memory
}

\author{
Vivek Srivastava \\ Teacher Fellow \\ Department of Computer Science \& \\ Engineering \\ Harcourt Butler Technological \\ Institute, Kanpur INDIA
}

\author{
Vinay kumar Pathak \\ Professor \\ Department of Computer Science \& \\ Engineering \\ Harcourt Butler Technological \\ Institute, Kanpur INDIA
}

\begin{abstract}
Automated Human recognition is challenging area to identify the human based on characteristics such as behavioral or physical. In this paper we propose a noble approach for identifying the human by combining the two biometric face and ear recognition through bidirectional associative memory. This approach can be more suitable in passive environment such as surveillance .By combining two modality, we get the comparatively lower values of false rejection rate (FRR) and falsee acceptance rate (FAR). Using the proposed approach we can get comparatively lower value of FAR.
\end{abstract}

\section{Categories and Subject Descriptors}

[Neural Computing]: Bidirectional associative memory

\section{General Terms}

Algorithms, Security, Verification.

\section{Keywords}

Multimodal Biometrics, Face, Ear Recognition, Bidirectional associative memory (BAM).

\section{INTRODUCTION}

Earlier there exist many algorithms which identify the human based on their physical and behavioral characteristics. Human face detection is the first crucial step for human face recognition. In the recent years face detection and recognition problem is of great concern [3]-[5]. Fully automated human face recognition system is currently a challenging task. we can easily detects and recognize the human but it is difficult for computers to recognize the identity of the human. Human recognition are more difficult in a non co operational environment such as surveillance. In this situation face and ear are such biometric which can be captured easily by the cameras. On combining these two modalities we can get the higher recognition rate. Chang [8] identifies the advantages of combining face and ear biometric, using PCA for recognition.

\section{BACKGROUND}

\subsection{Face Recognition}

Face recognition is a form of biometric identification. There exist many face identification methods which have been proposed in last decades. Principal component analysis(PCA) is the one of the tool for extracting the feature from the images[5]. One of the earliest works in face detection was reported by Sakai et. al. [10]. They define the model of a human face in terms of several sub templates corresponding to face contour, eye, nose, and mouth. An edge map extracted from the input image is matched against the sub templates with possible variations in the position and size.

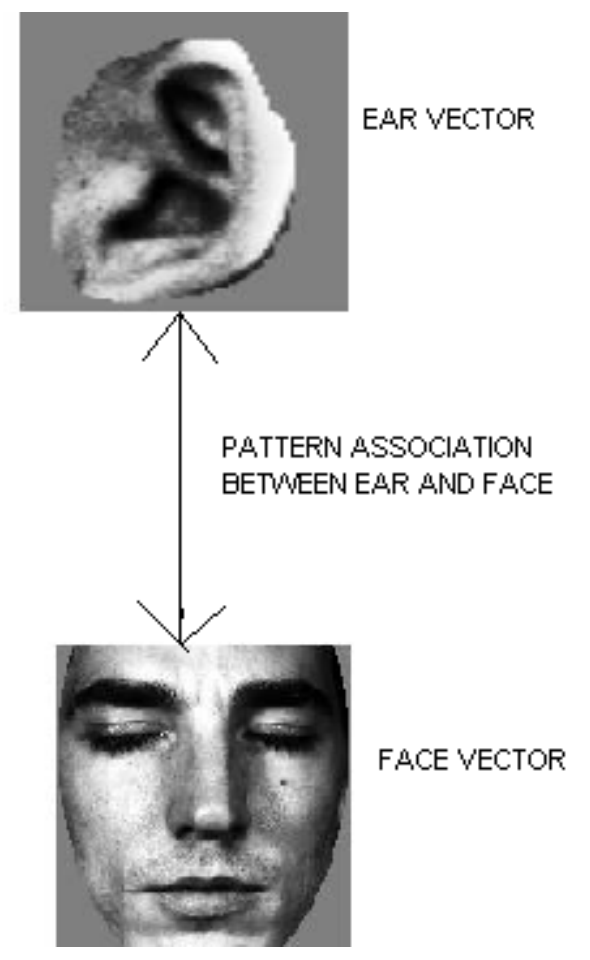

Fig-1. Representing the pattern association between ear and face. 


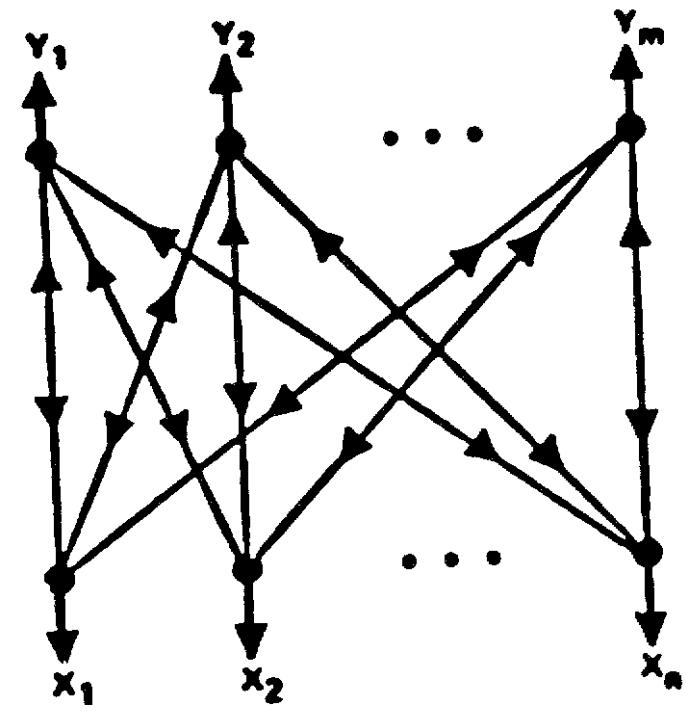

Fig. 2. Representation of BAM having $(\mathrm{x} 1, \mathrm{x} 2 \ldots . . \mathrm{xn})$ at one layer which maps to the $(\mathrm{y} 1, \mathrm{y} 2 \ldots . . . \mathrm{ym})$ at another layer .

\subsection{Ear Recognition}

Ear biometric is very useful for identifying the people of it's robustness. shape of ear doesn't changes and remains constant on facial expression however uniqueness of ear is still matter of discussion .but there are some evidence that proves ears are unique enough to be used as biometric.

Most famous work is made by Alfred Iannarelli at 1989 for uniqueness of ear, when he collected over 10,000 ears and found that they all were different. Ear biometric is not commonly used but it is especially used for crime investigation.

\subsection{Bidirectional Associative Memory}

Bidirectional associative memory was introduced by Bart Kosko[1]..BAM behaves as a hetero associative content addressable memory(CAM) storing and recalling vector pairs $(\mathrm{A} 1, \mathrm{~B} 1) \ldots \ldots . .(\mathrm{Am}, \mathrm{Bm})$ where $\mathrm{A} \epsilon\{0,1\}$ and $\mathrm{B} \epsilon\{0,1\} . \mathrm{BAM}$ is a kind of neural network having two layer $\mathrm{X}$-layer and $\mathrm{Y}$ - layer having set of connections from each input to output. It is a kind of neural network having two layers connected with each other.Also there is feedback from output layer to input layer(fig2).

Kohonen was the first to use an auto associative memory to store and recall face image [11]-[12]. He demonstrated that an auto associative memory could act as a content addressable memory for face image. In this paper we use two auto associative memories to validate candidate faces.

\section{PROPOSED ALGORITHM}

We propose the two phase algorithm to identify the human by capturing the two biometric face and ear. face and ear are the such two biometric that can be captured by the camera in passive environment .Individually face recognition algorithm in general gives the higher FRR as compared to FAR. while ear recognition algorithm can produce comparatively low value of FRR and FAR. By combining these two biometric we can get the comparatively lower value of FRR as well as FAR. We suggest two phase algorithm in which first phase involves the feature extraction of the face and ear biometric and training of the two biometric using bi directional associative memory in which the patterns of face and ear are associated to each other. In the second phase we test the new face using the BAM. On the basis of stored vector of the face and ear we match the test face and ear with the existing one. there are four possibilities for matching. first if new face matches with existing one but new ear doesn't matches .second new ear matches but new test face doesn't matches .third if both new face and ear matches. forth and last case if none of face or ear matches with existing one. based on these four possibilities we fuse the information at decision level .

On fusion of these information at decision level, we can improve the false acceptance rate(FAR) and false rejection rate (FRR).

\subsection{Phase One: Training}

First phase of proposed approach involves the following steps1. Input the set of faces and respective ears with their identity.

2. Extract the features of faces and respective ears using existing algorithm.

3. Construct the vector space for faces and respective ears.

4. Map the vectors of faces to vectors of their respective ears using the bidirectional associative memory having face vector $[\mathrm{x}]$ at one side and ear vector $[\mathrm{y}]$ at another side. compute the weight matrix for association of face vector with respective ear vector.

\subsection{Phase Two: Testing}

In this phase we test the new faces and ears based on our existing trained associated pattern of the faces and ears. this phase involves the following steps-

1. Extract the features of new test face and ear.

2. Map this new face vector with the new test ear using BAM.

3. Compute the weight matrix for association of new test face with test ear.

4. Match the computed matrix with existing matrices. Take the decision according to the matching score.

Let the face vector is represented by $\mathrm{F}$ and ear vector by $\mathrm{E}$ that is obtained after the feature extraction. We can compute the weight matrix $\mathrm{W}$ using BAM as following -

$$
\mathrm{E}=\mathrm{F} * \mathrm{~W}
$$

Then vector $\mathrm{E}$ and $\mathrm{F}$ can be associated using equation (1 )or the following equation

$$
\mathrm{F}=\mathrm{E} * \mathrm{~W}^{\mathrm{T}}
$$

For testing the new face vector F1 and new ear vector E1 we compute the weight matrix using equation (1) and (2).based on the result ,if F1 associated with $\mathrm{E} 1$ then we recognize the human 
by which F1 and E1 has been obtained . Basically above approach follow the conjunction rule or AND rule by which we accept only those people if both the biometric traits are matched. In this method false accept will occur if both $\mathrm{F} 1$ and $\mathrm{E} 1$ produce a false accept. This approach may be most useful under higher security level in which no false acceptantance is allowed .certainly proposed method comparatively reduces the FAR.

\section{CONCLUSION}

We propose the new approach for Human recognition by combining the two biometric face and ear through bidirectional associative memory. proposed approach can be useful for automatic passive human recognition system in which camera captures the ear and the face of the human in surveillance. This method employs less memory and fast training time as neural network takes less time comparative to other techniques.Multibiometric can improve performance through reducing the false acceptance rate(FAR) and false rejection rate(FRR).

\section{ACKNOWLEDGMENTS}

Biometric and Image processing research at Harcourt Butler Technological Institute (HBTI) is supported by department of computer science \& engineering, HBTI and IIT Kanpur.

\section{REFERENCES}

[1] Bart kosko, Bidirectional associative memory, IEEE transactions on system man and cybernatics vol 18 no. 1,1988

[2] T. Sakai, M. Nagao, and S. Fujibayashi, "Line extraction and pattern recognition in Marchionini, photograph, "Patt. Recog., vol. 1, pp. 233-248, 1969.
[3] R. Brunelli and T. Poggio, "Face recognition: Features versus templates," IEEE Trans. Patt. Anal. And Mach .Intell., vol. 15, pp. 1042-1052, 1993.

[4] M. Kirby and L. Sirovich, "Application of theKarhunenLoeve procedure for the characterization of human face,"MIEEE trans. Patt. Anal. And Mach. Intell.,vol. 12, pp. 103-108, 1990.

[5] M. Turk and A. Pentland, "Eigenfaces for recognition, "Journal of Cognitive Neuroscience, vol. 3, no. 1,app.71-86, 1991.

[6] L. Harmon and W. hunt, "Automatic recognition of human face profiles, " Computer Graphic and Image Process ., vol. 6, pp. 135-156, 1997.

[7] T. Kanade, Computer Recognition of Human Faces. Basel and Stuttgart: Birkhauser, 1997.

[8] Y.T. Yu, M. K.chang, K. Bowyer, and Barnabas. Comparision and combination of ear and face images in appearance based biometric IEEE Transaction on Pattern Analysis and Machine Intelligence,25:1160-1165,2003.

[9] Burge, M. and Burger, W. Ear Biometrics. in Computer Vision. In the 15th International Conference of Pattern Recognition, ICPR 2000, pp. 826-830.

[10] T. Sakai, M. Nagao, and S. Fujibayashi, "Lineextraction and pattern recognition in a photograph," Patt. Recog., vol. 1, pp. 233-248, 1969.

[11] T. K. Kohonen, Associative Memory: A System TheoreticApproaches, Springer, Berlin, 1977.

[12] T. Kohonen, E. Oju, and P. Lehtio, "Storage and processing of information in distributed associative memory systems," Parallel Models of Associative Memory, G. E. Hinton and J. A. Anderson, editors, pp. 49-81. Erlbaum, Hillsdale, 1981. 